\title{
Resonance peak in underdoped cuprates
}

\author{
A. Sherman \\ Institute of Physics, University of Tartu, Riia 142, 51014 Tartu, Estonia \\ M. Schreiber \\ Institut für Physik, Technische Universität, D-09107 Chemnitz, Federal Republic of Germany
}

(Dated: October 31, 2018)

\begin{abstract}
The magnetic susceptibility measured in neutron scattering experiments in underdoped $\mathrm{YBa}_{2} \mathrm{Cu}_{3} \mathrm{O}_{7-y}$ is interpreted based on the self-consistent solution of the $t-J$ model of a Cu-O plane. The calculations reproduce correctly the frequency and momentum dependencies of the susceptibility and its variation with doping and temperature in the normal and superconducting states. This allows us to interpret the maximum in the frequency dependence - the resonance peak - as a manifestation of the excitation branch of localized $\mathrm{Cu}$ spins and to relate the frequency of the maximum to the size of the spin gap. The low-frequency shoulder well resolved in the susceptibility of superconducting crystals is connected with a pronounced maximum in the damping of the spin excitations. This maximum is caused by intense quasiparticle peaks in the hole spectral function for momenta near the Fermi surface and by the nesting.

PACS numbers: $71.10 . \mathrm{Fd}, 74.25 . \mathrm{Ha}$
\end{abstract}

\section{INTRODUCTION}

Inelastic neutron scattering experiments give important information on the anomalous properties of high- $T_{c}$ superconductors. Among the results obtained with this experimental method is the detailed information on the magnetic susceptibility in $\mathrm{YBa}_{2} \mathrm{Cu}_{3} \mathrm{O}_{7-y}$ measured in wide ranges of hole concentrations and temperatures $\frac{1.2}{1}$ These measurements revealed the sharp magnetic collective mode called the resonance peak. The peak first observed ${ }^{3.4}$ in the superconducting state of $\mathrm{YBa}_{2} \mathrm{Cu}_{3} \mathrm{O}_{7}$ was later also detected in the underdoped compounds, both in the superconducting and normal states ${ }^{2.5}$ Recently the resonance peak was also observed in $\mathrm{Bi}_{2} \mathrm{Sr}_{2} \mathrm{CaCu}_{2} \mathrm{O}_{8+\delta}$ and $\mathrm{Tl}_{2} \mathrm{Ba}_{2} \mathrm{CuO}_{6+\delta} \cdot \underline{6}$

Theoretical works devoted to the resonance peak were mainly concentrated at the overdoped region where the peak is observed in the superconducting state and disappears in the normal state 1.2 .3 .4 .5 .6 In Refs. 789 an interpretation of the resonance peak based on the itinerant magnetism approach was proposed. This approach which uses the Lindhard function for the bare susceptibility $\chi_{0}(\mathbf{k} \omega)$ and the random phase approximation relates the appearance of the peak to the disappearance or considerable decrease of $\operatorname{Im} \chi_{0}(\mathbf{Q} \omega)$ in the frequency range $\omega \leq 2 \Delta^{s}$ with the opening of the $d$-wave ${ }^{7.9}$ or $s$-wave w $^{8}$ superconducting gaps $\Delta^{s}$. Here $\mathbf{Q}=(\pi, \pi)$ is the antiferromagnetic wave vector. In this frequency range the peak arises due to the logarithmic divergence in $\operatorname{Re} \chi_{0}(\mathbf{Q} \omega)$

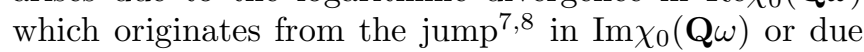
to the nesting of the bonding and antibonding Fermi surfaces in the two-layer crystal $\frac{9}{\underline{\underline{a}}}$ In the normal state, when the damping increases, the peak is smeared out. For the approaches of Refs. 8.9 the two-layer structure of $\mathrm{YBa}_{2} \mathrm{Cu}_{3} \mathrm{O}_{7-y}$ is of crucial importance for the appearance of the resonance peak. However, the recent observation ${ }^{6}$ of the peak in single-layer $\mathrm{Tl}_{2} \mathrm{Ba}_{2} \mathrm{CuO}_{6+\delta}$ indicates that an interaction between closely spaced $\mathrm{Cu}$ $\mathrm{O}$ layers is not the necessary condition. A single-layer system described by a modified $s-f$ Hamiltonian was considered in Ref. 10. In that work the resonance peak appears also due to the vanishing damping of spin excitations and a real part of the spin excitation frequency stems from the fermion bubble. A qualitatively different approach was suggested in Ref. 11 where the existence of a well-defined branch of spin excitations which exists even in the absence of mobile carriers was postulated near the $M$ point $(\mathbf{k}=\mathbf{Q})$ of the Brillouin zone. In this scenario the resonance peak is related to the excitation with $\mathbf{k}=\mathbf{Q}$ of this branch. As in Ref. 7, 89, here the peak is visible in the superconducting state due to the absence of the damping for $\omega \leq 2 \Delta^{s}$ and is smeared out in the normal state.

This latter scenario seems to correspond most adequately to available experimental data on the resonance peak. As mentioned, it is observed also in underdoped $\mathrm{YBa}_{2} \mathrm{Cu}_{3} \mathrm{O}_{7-y}$ and in the superconducting state the peak varies continuously on passing from the underdoped to overdoped region. Moreover, in the underdoped region the peak is also observed in the normal state and its frequency is nearly the same as in the superconducting state. Therefore it is reasonable to search for a unified explanation for the peak which is applicable both for underdoped and overdoped regions, and - in the former region - for the normal and superconducting states. The existence of the excitation branch of localized $\mathrm{Cu}$ spins is well established for the underdoped region ${ }^{2.12}$ In this region it is quite reasonable to connect the resonance peak with these excitations.

In this paper we use the two-dimensional $t-J$ model to which the realistic three-band Hubbard model of the $\mathrm{CuO}_{2}$ planes can be mapped in the case of a strong onsite Coulomb repulsion ${ }^{13}$ For the underdoped region the self-consistent solution of the $t-J$ model was obtained in 
Ref. 14 with the use of Mori's projection operator technique. We employ this result for the calculation of the magnetic susceptibility in the normal and superconducting states. The calculations reproduce correctly the frequency and momentum dependencies of the susceptibility and its evolution with doping and temperature in $\mathrm{YBa}_{2} \mathrm{Cu}_{3} \mathrm{O}_{7-y}$. This allows us to relate the resonance peak with the excitation branch of the localized $\mathrm{Cu}$ spins and to identify the frequency of the peak with the size of the spin gap at the $M$ point. In the underdoped region, with increasing doping the peak frequency grows with the gap size and the peak intensity decreases, in agreement with experimental observations, ${ }^{1.2}$ Moreover, the low-frequency shoulder observed ${ }^{1.2}$ in the susceptibility of superconducting crystals can be connected with a pronounced maximum which we find in the damping of the spin excitations. This maximum is caused by intense quasiparticle peaks in the hole spectral function for momenta near the Fermi surface and by the nesting.

\section{THE HOLE GREEN'S FUNCTION IN THE SUPERCONDUCTING STATE}

The Hamiltonian of the $2 \mathrm{D} t-J$ model reads 15

$$
H=\sum_{\mathbf{n} \mathbf{m} \sigma} t_{\mathbf{n} \mathbf{m}} a_{\mathbf{n} \sigma}^{\dagger} a_{\mathbf{m} \sigma}+\frac{1}{2} \sum_{\mathbf{n} \mathbf{m}} J_{\mathbf{n m}}\left(s_{\mathbf{n}}^{z} s_{\mathbf{m}}^{z}+s_{\mathbf{n}}^{+1} s_{\mathbf{m}}^{-1}\right)
$$

where $a_{\mathbf{n} \sigma}=|\mathbf{n} \sigma\rangle\langle\mathbf{n} 0|$ is the hole annihilation operator, $\mathbf{n}$ and $\mathbf{m}$ label sites of the square lattice, $\sigma= \pm 1$ is the spin projection, $|\mathbf{n} \sigma\rangle$ and $|\mathbf{n} 0\rangle$ are site states corresponding to the absence and presence of a hole on the site. These states are linear combinations of the products of the $3 d_{x^{2}-y^{2}}$ copper and $2 p_{\sigma}$ oxygen orbitals of the extended Hubbard model ${ }^{13}$ In this work we take into account nearest neighbor interactions only, $t_{\mathbf{n m}}=-t \sum_{\mathbf{a}} \delta_{\mathbf{n}, \mathbf{m}+\mathbf{a}}$ and $J_{\mathbf{n m}}=J \sum_{\mathbf{a}} \delta_{\mathbf{n}, \mathbf{m}+\mathbf{a}}$ where the four vectors a connect nearest neighbor sites. The spin- $\frac{1}{2}$ operators can be written as $s_{\mathbf{n}}^{z}=\frac{1}{2} \sum_{\sigma} \sigma|\mathbf{n} \sigma\rangle\langle\mathbf{n} \sigma|$ and $s_{\mathbf{n}}^{\sigma}=|\mathbf{n} \sigma\rangle\langle\mathbf{n},-\sigma|$.

To investigate the magnetic susceptibility of this model the hole $\mathbf{G}(\mathbf{k} t)=-i \theta(t)\left\langle\left\{\mathbf{A}_{\mathbf{k} \sigma}(t), \mathbf{A}_{\mathbf{k} \sigma}^{\dagger}\right\}\right\rangle$ and spin $D(\mathbf{k} t)=-i \theta(t)\left\langle\left[s_{\mathbf{k}}^{z}(t), s_{-\mathbf{k}}^{z}\right]\right\rangle$ Green's functions have to be calculated. Here supposing the singlet superconducting pairing we introduced the Nambu spinor,

$$
\mathbf{A}_{\mathbf{k} \sigma}=\left(\begin{array}{c}
a_{\mathbf{k} \sigma} \\
a_{-\mathbf{k},-\sigma}^{\dagger}
\end{array}\right)
$$

thus $\mathbf{G}$ is a $2 \times 2$ matrix (here and below matrices and vectors are designated by boldface letters). In the above formulas the angular brackets denote averaging over the grand canonical ensemble, and

$$
\begin{aligned}
a_{\mathbf{k} \sigma} & =N^{-1 / 2} \sum_{\mathbf{n}} e^{-i \mathbf{k n}} a_{\mathbf{n} \sigma} \\
s_{\mathbf{k}}^{z} & =N^{-1 / 2} \sum_{\mathbf{n}} e^{-i \mathbf{k} \mathbf{n}} s_{\mathbf{n}}^{z},
\end{aligned}
$$

$a_{\mathbf{k} \sigma}(t)=\exp (i \mathcal{H} t) a_{\mathbf{k} \sigma} \exp (-i \mathcal{H} t), N$ is the number of sites, $\mathcal{H}=H-\mu \sum_{\mathbf{n}} X_{\mathbf{n}}, \mu$ is the chemical potential, $X_{\mathbf{n}}=|\mathbf{n} 0\rangle\langle\mathbf{n} 0|$.

To derive the self-energy equation for the matrix Green's function G the continued fraction representation for Green's function and the recursive equations for their elements from Ref. 14 have to be generalized for the case of matrices. Such generalization reads

$$
\mathbf{R}_{n}(\omega)=\left[\omega \mathbf{I}-\mathbf{E}_{n}-\mathbf{R}_{n+1}(\omega) \mathbf{F}_{n}\right]^{-1}, \quad n=0,1,2 \ldots
$$

where $\mathbf{I}$ is a $2 \times 2$ unit matrix, the matrices $\mathbf{E}_{n}$ and $\mathbf{F}_{n}$ are calculated from the recursive equations

$$
\begin{aligned}
& {\left[\mathbf{A}_{n}, H\right]=\mathbf{E}_{n} \mathbf{A}_{n}+\mathbf{A}_{n+1}+\mathbf{F}_{n-1} \mathbf{A}_{n-1},} \\
& \mathbf{E}_{n}=\left\langle\left\{\left[\mathbf{A}_{n}, H\right], \mathbf{A}_{n}^{\dagger}\right\}\right\rangle\left\langle\left\{\mathbf{A}_{n}, \mathbf{A}_{n}^{\dagger}\right\}\right\rangle^{-1}, \\
& \mathbf{F}_{n}=\left\langle\left\{\mathbf{A}_{n+1}, \mathbf{A}_{n+1}^{\dagger}\right\}\right\rangle\left\langle\left\{\mathbf{A}_{n}, \mathbf{A}_{n}^{\dagger}\right\}\right\rangle^{-1} .
\end{aligned}
$$

Here $\mathbf{F}_{-1}=0$ and $\mathbf{A}_{0}=\mathbf{A}_{\mathbf{k} \sigma}$ for the case of the function G. As follows from Eq. (3), the two-component operators $\mathbf{A}_{n}$ constructed in this recursive procedure form an orthogonal basis. For the anticommutator Green's function $\mathbf{G}$ the inner product of two arbitrary operators $\mathbf{A}$ and $\mathbf{B}$ is defined as $\langle\{\mathbf{A}, \mathbf{B}\}\rangle$ and the orthogonality means $\left\langle\left\{\mathbf{A}_{n}, \mathbf{A}_{m}\right\}\right\rangle=\delta_{n m}\left\langle\left\{\mathbf{A}_{n}, \mathbf{A}_{n}\right\}\right\rangle$. In Eq. (2), $\quad \mathbf{R}_{n}(\omega)=-i \int_{0}^{\infty} d t \exp (i \omega t) \mathbf{R}_{n}(t), \quad \mathbf{R}_{n}(t)=$ $\left\langle\left\{\mathbf{A}_{n t}, \mathbf{A}_{n}^{\dagger}\right\}\right\rangle\left\langle\left\{\mathbf{A}_{n}, \mathbf{A}_{n}^{\dagger}\right\}\right\rangle^{-1}$ where the time dependencies are determined by the equation

$$
i \frac{d}{d t} \mathbf{A}_{n t}=\prod_{k=0}^{n-1}\left(1-P_{k}\right)\left[\mathbf{A}_{n t}, H\right], \quad \mathbf{A}_{n, t=0}=\mathbf{A}_{n}
$$

with the definition $P_{n} \mathbf{Q}=\left\langle\left\{\mathbf{Q}, \mathbf{A}_{n}^{\dagger}\right\}\right\rangle\left\langle\left\{\mathbf{A}_{n}, \mathbf{A}_{n}^{\dagger}\right\}\right\rangle^{-1} \mathbf{A}_{n}$ of the projection operator $P_{n}$ that projects an arbitrary two-component operator $\mathbf{Q}$ on $\mathbf{A}_{n}$ (for a more detailed discussion of these equations see Ref. 14).

From the above definitions it follows for the Fourier transformation of $\mathbf{G}(\mathbf{k} t)$

$$
\mathbf{G}(\mathbf{k} \omega)=\left[\omega \mathbf{I}-\mathbf{E}_{0}-\mathbf{R}_{1} \mathbf{F}_{0}\right]^{-1}\left\langle\left\{\mathbf{A}_{0}, \mathbf{A}_{0}^{\dagger}\right\}\right\rangle,
$$

where $\left\langle\left\{\mathbf{A}_{0}, \mathbf{A}_{0}^{\dagger}\right\}\right\rangle=\varphi \mathbf{I}, \varphi=\frac{1}{2}(1+x), x=\left\langle X_{\mathbf{n}}\right\rangle$ is the hole concentration,

$$
\mathbf{E}_{0}=\left(\begin{array}{cc}
\varepsilon_{\mathbf{k}}-\mu^{\prime} & \sigma K_{1} \varphi^{-1}\left(3 J \gamma_{\mathbf{k}}-8 t\right) \\
\sigma K_{1}^{*} \varphi^{-1}\left(3 J \gamma_{\mathbf{k}}-8 t\right) & -\left(\varepsilon_{\mathbf{k}}-\mu^{\prime}\right)
\end{array}\right)
$$

$\varepsilon_{\mathbf{k}}=-\left(4 t \varphi+6 t C_{1} \varphi^{-1}+3 J F_{1} \varphi^{-1}\right) \gamma_{\mathbf{k}}, \mu^{\prime}=\mu+4 t F_{1} \varphi^{-1}+$ $3 J C_{1} \varphi^{-1}, \gamma_{\mathbf{k}}=\frac{1}{4} \sum_{\mathbf{a}} \exp (i \mathbf{k a})$. The nearest-neighbor correlations $C_{1}=\left\langle s_{\mathbf{n}}^{+1} s_{\mathbf{n}+\mathbf{a}}^{-1}\right\rangle, F_{1}=\left\langle a_{\mathbf{n} \sigma}^{\dagger} a_{\mathbf{n}+\mathbf{a}, \sigma}\right\rangle, K_{1}=$ $\sigma\left\langle a_{\mathbf{n} \sigma} a_{\mathbf{n}+\mathbf{a},-\sigma}\right\rangle$ and the hole concentration $x$ can be expressed in terms of the components of the hole and spin Green's functions:

$$
\begin{aligned}
x & =\frac{1}{N} \sum_{\mathbf{k}} \int_{-\infty}^{\infty} d \omega n_{F}(\omega) A(\mathbf{k} \omega), \\
F_{1} & =\frac{1}{N} \sum_{\mathbf{k}} \gamma_{\mathbf{k}} \int_{-\infty}^{\infty} d \omega n_{F}(\omega) A(\mathbf{k} \omega),
\end{aligned}
$$




$$
\begin{aligned}
C_{1}= & \frac{2}{N} \sum_{\mathbf{k}} \gamma_{\mathbf{k}} \int_{0}^{\infty} d \omega \operatorname{coth}\left(\frac{\omega}{2 T}\right) B(\mathbf{k} \omega), \\
K_{1}= & \frac{\sigma}{N} \sum_{\mathbf{k}} \gamma_{\mathbf{k}} \int_{-\infty}^{\infty} d \omega\left[1-n_{F}(\omega)\right] \\
& \times[L(\mathbf{k} \omega \sigma)+i M(\mathbf{k} \omega \sigma)],
\end{aligned}
$$

where

$$
\begin{aligned}
L(\mathbf{k} \omega \sigma) & =-\operatorname{Im}\left[G_{12}(\mathbf{k} \omega \sigma)+G_{21}(\mathbf{k} \omega \sigma)\right] /(2 \pi), \\
M(\mathbf{k} \omega \sigma) & =\operatorname{Re}\left[G_{12}(\mathbf{k} \omega \sigma)-G_{21}(\mathbf{k} \omega \sigma)\right] /(2 \pi),
\end{aligned}
$$

$A(\mathbf{k} \omega)=-\operatorname{Im} G_{11}(\mathbf{k} \omega) / \pi$, and $B(\mathbf{k} \omega)=-\operatorname{Im} D(\mathbf{k} \omega) / \pi$ are the hole and spin spectral functions, $n_{F}(\omega)=$ $[\exp (\omega / T)+1]^{-1}$ and $T$ is the temperature (the functions $A(\mathbf{k} \omega)$ and $B(\mathbf{k} \omega)$ do not depend on $\sigma)$. In the derivation of Eq. (6) for $C_{1}$ we have taken into account that the approximation used retains the rotation symmetry of spin components ${ }^{14}$ and therefore $C_{1}=2\left\langle s_{\mathbf{n}}^{z} s_{\mathbf{n}+\mathbf{a}}^{z}\right\rangle$.

As follows from Eq. (5), in the $t-J$ model the superconducting gap has an $s$-wave component if $K_{1} \neq 0$. However, in the considered case this component is small in comparison with the $d$-wave component introduced below and will be neglected.

From the definition of the hole self-energy $\boldsymbol{\Sigma}=\mathbf{R}_{\mathbf{1}} \mathbf{F}_{\mathbf{0}}$ we find that $\Sigma_{22}(\mathbf{k} \omega)=\Sigma_{11}^{*}(\mathbf{k},-\omega)$ where it was taken into account that these components of $\boldsymbol{\Sigma}$ do not depend on $\sigma$ and are invariant under the inversion of $\mathbf{k}$. For $\Sigma_{11}(\mathbf{k} \omega)$ the following expression obtained in Ref. 14 can be used:

$$
\begin{aligned}
& \operatorname{Im} \Sigma_{11}(\mathbf{k} \omega)=\frac{16 \pi t^{2}}{N \phi} \sum_{\mathbf{k}^{\prime}} \int_{-\infty}^{\infty} d \omega^{\prime}\left[\gamma_{\mathbf{k}-\mathbf{k}^{\prime}}+\gamma_{\mathbf{k}}\right. \\
& \left.+\operatorname{sgn}\left(\omega^{\prime}\right)\left(\gamma_{\mathbf{k}-\mathbf{k}^{\prime}}-\gamma_{\mathbf{k}}\right) \sqrt{\frac{1+\gamma_{\mathbf{k}^{\prime}}}{1-\gamma_{\mathbf{k}^{\prime}}}}\right]^{2} \\
& \times\left[n_{B}\left(-\omega^{\prime}\right)+n_{F}\left(\omega-\omega^{\prime}\right)\right] \\
& \times A\left(\mathbf{k}-\mathbf{k}^{\prime}, \omega-\omega^{\prime}\right) B\left(\mathbf{k}^{\prime} \omega^{\prime}\right) \text {, } \\
& \operatorname{Re} \Sigma_{11}(\mathbf{k} \omega)=\mathcal{P} \int_{-\infty}^{\infty} \frac{d \omega^{\prime}}{\pi} \frac{\operatorname{Im} \Sigma_{11}\left(\mathbf{k} \omega^{\prime}\right)}{\omega^{\prime}-\omega},
\end{aligned}
$$

where $n_{B}(\omega)=[\exp (\omega / T)-1]^{-1}$ and $\mathcal{P}$ indicates Cauchy's principal value.

Assuming the $d$-wave superconducting pairing, for the anomalous self-energies we set

$$
\Sigma_{12}(\mathbf{k} \omega \sigma)=\Sigma_{12}(\mathbf{k} \omega \sigma)=\sigma \Delta^{s}\left[\cos \left(k_{x}\right)-\cos \left(k_{y}\right)\right] / 2,
$$

with the superconducting gap $\Delta^{s}$. For such anomalous self-energies $M(\mathbf{k} \omega \sigma)=0$.

\section{THE SPIN GREEN'S FUNCTION}

In Ref. 14 we have noticed that the approximation used there leads to an underestimation of the imaginary part of the magnetic susceptibility at low frequencies. To avoid this drawback in the present work we shall not split the spin self-energy into the hole and spin parts, but rather continue the calculation of the terms of the continued fraction using the entire Hamiltonian (11).

The spin Green's function is calculated from the relation

$$
D(\mathbf{k} \omega)=\omega\left(\left(s_{\mathbf{k}}^{z} \mid s_{-\mathbf{k}}^{z}\right)\right)_{\omega}-\left(s_{\mathbf{k}}^{z}, s_{-\mathbf{k}}^{z}\right)
$$

where

$$
\left(s_{\mathbf{k}}^{z}, s_{-\mathbf{k}}^{z}\right)=i \int_{0}^{\infty} d t\left\langle\left[s_{\mathbf{k}}^{z}(t), s_{-\mathbf{k}}^{z}\right]\right\rangle,
$$

and Kubo's relaxation function

$$
\left(\left(s_{\mathbf{k}}^{z} \mid s_{-\mathbf{k}}^{z}\right)\right)_{\omega}=\int_{0}^{\infty} d t e^{i \omega t} \int_{t}^{\infty} d t^{\prime}\left\langle\left[s_{\mathbf{k}}^{z}\left(t^{\prime}\right), s_{-\mathbf{k}}^{z}\right]\right\rangle
$$

can be represented by a continued fraction which is similar to the scalar form of Eq. (2). The elements $E_{n}$ and $F_{n}$ of this function are calculated from a recursive procedure which is similar to the scalar form of Eq. (3) where, however, mean values of anticommutators have to be substituted by inner products of the type of Eq. (10) (see Ref. 14).

From this definition we find for the starting operator $A_{0}=s_{\mathbf{k}}^{z}$ of this recursive procedure

$$
E_{0}=\left(i \dot{s}_{\mathbf{k}}^{z}, s_{-\mathbf{k}}^{z}\right)\left(s_{\mathbf{k}}^{z}, s_{-\mathbf{k}}^{z}\right)^{-1}=0
$$

where $i \dot{s}_{\mathbf{k}}^{z}=\left[s_{\mathbf{k}}^{z}, H\right]$,

$$
A_{1}=i \dot{s}_{\mathbf{k}}^{z}, \quad F_{0}=\frac{4\left(1-\gamma_{\mathbf{k}}\right)\left(J\left|C_{1}\right|+t F_{1}\right)}{\left(s_{\mathbf{k}}^{z}, s_{-\mathbf{k}}^{z}\right)}, \quad E_{1}=0
$$

Using these elements of the continued fraction representation of $\left(\left(s_{\mathbf{k}}^{z} \mid s_{-\mathbf{k}}^{z}\right)\right)_{\omega}$, Eq. (9) can be rewritten as

$$
D(\mathbf{k} \omega)=\frac{4\left(1-\gamma_{\mathbf{k}}\right)\left(J\left|C_{1}\right|+t F_{1}\right)}{\omega^{2}-\omega \Pi(\mathbf{k} \omega)-\omega_{\mathbf{k}}^{2}}
$$

where $\omega_{\mathbf{k}}^{2}=F_{0}$,

$$
\begin{aligned}
\Pi(\mathbf{k} \omega)= & -i\left[4\left(1-\gamma_{\mathbf{k}}\right)\left(J\left|C_{1}\right|+t F_{1}\right)\right]^{-1} \\
& \times \int_{0}^{\infty} d t e^{i \omega t}\left(A_{2 t}, A_{2}^{\dagger}\right), \\
A_{2}= & i^{2} \ddot{s}_{\mathbf{k}}^{z}-\omega_{\mathbf{k}}^{2} s_{\mathbf{k}}^{z} .
\end{aligned}
$$

As follows from the above equation, to calculate $\omega_{\mathbf{k}}^{2}$ and $A_{2}$ we have to select terms of $i^{2} \ddot{s}_{\mathbf{k}}^{z}$ which are proportional to $s_{\mathbf{k}}^{z}$. It can be done only approximately because the quantity $\left(s_{\mathbf{k}}^{z}, s_{-\mathbf{k}}^{z}\right)$ cannot be calculated exactly. Following Refs. 14,16 we used the decoupling in $i^{2} \ddot{s}_{\mathbf{k}}^{z}$ for such selection and found

$$
\omega_{\mathbf{k}}^{2}=16 \alpha J^{2}\left(\left|C_{1}\right|+\frac{t F_{1}}{\alpha J}\right)\left(1-\gamma_{\mathbf{k}}\right)\left(\Delta+1+\gamma_{\mathbf{k}}\right),
$$

where $\Delta$ is the parameter of the gap in the spin excitation spectrum at the wave vector $\mathbf{Q}$ of the Brillouin zone. In an infinite 2D lattice this gap is opened for any 
nonzero temperature ${ }^{16}$ and at $T=0$ for $x \gtrsim 0.02 .14$ The gap size is directly connected with the spin correlation length of the short-range antiferromagnetic order. Hence a finite gap for $T>0$ is in agreement with the MerminWagner theorem ${ }^{17}$ The gap parameter can be expressed through the model parameters and correlations of hole and spin operators. ${ }^{14.16}$ However, due to strong dependencies of the considered quantities on this parameter we found it more accurate to determine this parameter from the constraint of zero site magnetization $\left\langle s_{\mathbf{k}}^{z}\right\rangle=0$ which is fulfilled in the paramagnetic state. This constraint can be written in the form

$$
\frac{1}{2}(1-x)=\frac{2}{N} \sum_{\mathbf{k}} \int_{0}^{\infty} d \omega \operatorname{coth}\left(\frac{\omega}{2 T}\right) B(\mathbf{k} \omega) .
$$

In Eq. (13), the parameter $\alpha$ is introduced to improve somewhat the results obtained with the decoupling and to take into account vertex corrections. In earlier works 16 where the analogous correction were used for the Heisenberg model this parameter was determined from the constraint (14). Due to comparatively weak dependencies of the considered quantities on this parameter we found it more appropriate to set $\alpha=1.802-0.802 \tanh (10 x)$ and to use the constraint for the calculation of $\Delta$, as mentioned above. The expression given for $\alpha$ takes into account its value obtained in Ref. 14 for finite damping of spin excitations and the weakening of the vertex corrections with doping.

When selecting terms of $i^{2} \ddot{s}_{\mathbf{k}}^{z}$ which have to be included into $A_{2}$ we omitted terms proportional to $t^{2}$, being motivated by our earlier result ${ }^{14}$ and by the results of the spinwave approximation ${ }^{18}$ which indicate that $\Pi(\mathbf{k} \omega)$ has to be proportional to $t^{2}$. An additional argument to omit these terms is that a part of them contains multipliers of the type $\sum_{\sigma} \sigma a_{\mathbf{m} \sigma}^{\dagger} a_{\mathbf{m} \sigma}$ the mean values of which are zero. Other terms of this type and a part of terms proportional to $t J$ contain the hole operators with opposite spins, $a_{\mathbf{m} \sigma}^{\dagger} a_{\mathbf{m}^{\prime},-\sigma}$, which also give zero on averaging and therefore were omitted. Terms which are proportional to $J^{2}$ and describe multiple spin-excitation scattering processes were not included into $A_{2}$ either - in this article only the decay of the spin excitation into the fermion pair is considered. This process is described by the following terms:

$$
\begin{aligned}
A_{2}= & \frac{4 t J}{N} \sum_{\mathbf{k}_{1} \mathbf{k}_{2} \sigma} g_{\mathbf{k}_{1} \mathbf{k}_{2}} \overline{a_{\mathbf{k}_{1} \sigma}^{\dagger} a_{\mathbf{k}+\mathbf{k}_{1}-\mathbf{k}_{2}, \sigma} s_{\mathbf{k}_{2}}^{z}}, \\
g_{\mathbf{k}_{\mathbf{k}_{1} \mathbf{k}_{2}}=} & \left(\gamma_{\mathbf{k}_{2}}+\frac{1}{4}\right) \\
& \times\left(\gamma_{\mathbf{k}_{2}-\mathbf{k}_{1}}-\gamma_{\mathbf{k}_{1}}-\gamma_{\mathbf{k}+\mathbf{k}_{1}-\mathbf{k}_{2}}+\gamma_{\mathbf{k}+\mathbf{k}_{1}}\right),
\end{aligned}
$$

where the line over the operators indicates that in calculating thermodynamic averages with $A_{2}$ by factorization, terms containing couplings of hole operators from the same $A_{2}$ have to be omitted, since such processes have already been included into $\omega_{\mathbf{k}}^{2} s$. Substituting $A_{2}$ into the above definition of $\Pi(\mathbf{k} \omega)$, neglecting the difference between $A_{2 t}$ and $A_{2}(t)$ and using the decoupling we get

$$
\begin{aligned}
\operatorname{Im} \Pi(\mathbf{k} \omega) & =\frac{8 \pi t^{2} J^{2}}{N^{2}\left(1-\gamma_{\mathbf{k}}\right)\left(J\left|C_{1}\right|+t F_{1}\right)} \frac{1-\exp (\omega / T)}{\omega} \\
& \times \sum_{\mathbf{k}_{1} \mathbf{k}_{2}} g_{\mathbf{k} \mathbf{k}_{1} \mathbf{k}_{2}}^{2} \iint_{-\infty}^{\infty} d \omega_{1} d \omega_{2} n_{B}\left(\omega_{2}\right) \\
& \times\left[1-n_{F}\left(\omega_{1}\right)\right] n_{F}\left(\omega+\omega_{1}-\omega_{2}\right) B\left(\mathbf{k}_{2} \omega_{2}\right) \\
& \times\left[A\left(\mathbf{k}_{1} \omega_{1}\right) A\left(\mathbf{k}+\mathbf{k}_{1}-\mathbf{k}_{2}, \omega+\omega_{1}-\omega_{2}\right)\right. \\
& \left.-L\left(\mathbf{k}_{1} \omega_{1} \sigma\right) L\left(\mathbf{k}+\mathbf{k}_{1}-\mathbf{k}_{2}, \omega+\omega_{1}-\omega_{2}, \sigma\right)\right] .
\end{aligned}
$$

Equation (13) is supposed to give a good approximation for the real part of the frequency of spin excitations and therefore only the imaginary part of $\Pi(\mathbf{k} \omega)$ will be considered below. Notice that $\operatorname{Im} \Pi(\mathbf{k} \omega)$ is negative, finite for $\omega=0$ and even with respect to the change of the sign of $\omega$.

As seen from Eq. (15), $\operatorname{Im} \Pi(\mathbf{k} \omega)$ is finite for $\mathbf{k} \rightarrow 0$, whereas $\omega_{\mathbf{k}}$ vanishes in this limit. Therefore the spin Green's function (12) has a purely imaginary, diffusive pole near the $\Gamma$ point, in compliance with the result of the hydrodynamic theory ${ }^{19}$ In the general case properties of spin excitations near the $M$ point differ essentially from those near $\Gamma$. In the calculations of Ref. 14 for the former excitations the real parts of frequencies were larger than their imaginary parts due to the spin gap. However, it is worth noting that in this comparison only the decay into two fermions was considered as the source of damping. Another source of damping - multiple spin-excitation scattering - was neglected. However, even in the case of overdamped excitations with $\mathbf{k} \approx \mathbf{Q}$ their frequencies will have real components due to the spin gap.

To simplify further calculations we take into account that in the considered underdoped case the spin spectral function $B(\mathbf{k} \omega)$ is strongly peaked near $\mathbf{Q}$ for $\omega \approx \omega_{\mathbf{Q}}$. Allowing for the small value of $\omega_{\mathbf{Q}}, A(\mathbf{k}+\mathbf{Q}, \omega) \approx A(\mathbf{k} \omega)$ and Eq. (14) we get

$$
\begin{aligned}
\operatorname{Im} \Pi(\mathbf{k} \omega)= & \frac{9 \pi t^{2} J^{2}(1-x)}{2 N\left(1-\gamma_{\mathbf{k}}\right)\left(J\left|C_{1}\right|+t F_{1}\right)} \\
& \times \sum_{\mathbf{k}^{\prime}}\left(\gamma_{\mathbf{k}+\mathbf{k}^{\prime}}-\gamma_{\mathbf{k}^{\prime}}\right)^{2} \\
& \times \int_{-\infty}^{\infty} d \omega^{\prime} \frac{n_{F}\left(\omega+\omega^{\prime}\right)-n_{F}\left(\omega^{\prime}\right)}{\omega} \\
& \times\left[A\left(\mathbf{k}^{\prime} \omega^{\prime}\right) A\left(\mathbf{k}+\mathbf{k}^{\prime}, \omega+\omega^{\prime}\right)\right. \\
& \left.-L\left(\mathbf{k}^{\prime} \omega^{\prime} \sigma\right) L\left(\mathbf{k}+\mathbf{k}^{\prime}, \omega+\omega^{\prime}, \sigma\right)\right] .
\end{aligned}
$$

Now the damping has taken the familiar form given by the fermion bubble. 


\section{MAGNETIC SUSCEPTIBILITY}

We have used hole self-energies (7) and correlations of hole and spin operators obtained in Ref. 14 for calculating the hole Green's function G, Eq. (4). This function and the spin gap parameters $\Delta$ obtained in Ref. 14 have then been applied for the calculation of the spin Green's function determined by Eqs. (12), (13) and (16). This latter function is connected with the magnetic susceptibility by the relation

$$
\chi^{z}(\mathbf{k} \omega)=-4 \mu_{B}^{2} D(\mathbf{k} \omega)
$$

where $\mu_{B}$ is the Bohr magneton. The self-energies of Ref. 14 were calculated for a $20 \times 20$ lattice with the parameters $t=0.5 \mathrm{eV}, J=0.1 \mathrm{eV}$ which correspond to hole-doped cuprates ${ }^{13.20}$ and for the ranges of hole concentrations and temperatures $0 \leq x \leq 0.16$ and $0.01 t \approx 58 \mathrm{~K} \leq T \leq 0.2 t \approx 1200 \mathrm{~K}$. For several hole concentrations we have checked now that the self-energies calculated for $T=0.01 t$ remain practically unchanged as the temperature decreases to $T=0.003 t \approx 17 \mathrm{~K}$. Therefore we can use these self-energies also for $T<0.01 t$. For temperatures close to zero the superconducting gap $\Delta^{s}$ was set to $0.04 t=20 \mathrm{meV}$, the value extracted from the tunnelling experiments ${ }^{21}$ As follows from the experiments, this value remains practically unchanged with the doping variation from heavily underdoped to optimally doped $\mathrm{YBa}_{2} \mathrm{Cu}_{3} \mathrm{O}_{7-y}$.

Results of such calculations for the imaginary part of the magnetic susceptibility at the antiferromagnetic wave vector $\operatorname{Im} \chi(\mathbf{Q})$ are shown in Figs. 1] and 2 In these figures experimental data ${ }^{2}$ on the magnetic susceptibility of underdoped $\mathrm{YBa}_{2} \mathrm{Cu}_{3} \mathrm{O}_{7-y}$ are also depicted. The oxygen deficiencies $y=0.5$ and 0.17 in this crystal correspond to the hole concentrations $x \approx 0.075$ and $0.14 .^{22}$ $\mathrm{YBa}_{2} \mathrm{Cu}_{3} \mathrm{O}_{7-y}$ is a bilayer crystal and the symmetry allows one to divide the susceptibility into odd and even parts. For the antiferromagnetic intrabilayer coupling the odd part can be compared with our calculations carried out for a single layer.

The value of damping $|\operatorname{Im} \Pi(\mathbf{Q} \omega)|$ depends on widths of peaks in the hole spectral functions near the Fermi surface. These widths are determined by an artificial broadening which was introduced in Ref. 14 to stabilize the iteration procedure. From the comparison with photoemission spectra 23 of $\mathrm{YBa}_{2} \mathrm{Cu}_{3} \mathrm{O}_{7-y}$ it is seen that the peaks in Ref. 14 are more intensive and narrower than in experiment which leads to a larger value and stronger frequency dependence of the calculated damping. To weaken this difference and to obtain a better fit of the shapes of the calculated susceptibility to the experimental data we have decreased $|\operatorname{Im} \Pi(\mathbf{Q} \omega)|$ by a factor $f$ and added a constant damping $\eta$ to it. This allows us to weaken somewhat the frequency dependence of the total damping $\Gamma(\mathbf{Q} \omega)=|\operatorname{Im} \Pi(\mathbf{Q} \omega)| / f+\eta$. As will be discussed in greater details later, the low-frequency shoulder in $\operatorname{Im} \chi(\mathbf{Q} \omega)$ is connected with this dependence. Thus, the fitting parameters $f$ and $\eta$ allow us to change the
FIG. 1: The imaginary part of the spin susceptibility at the antiferromagnetic wave vector in the superconducting state. Curves show the results of our calculations in a $20 \times 20$ lattice for $t=0.5 \mathrm{eV}, J=0.1 \mathrm{eV}, T=17 \mathrm{~K}, x=0.06$ (a) and $x=0.12(\mathrm{~b})$. Filled squares are the odd susceptibility measured $^{2}$ in $\mathrm{YBa}_{2} \mathrm{Cu}_{3} \mathrm{O}_{6.5}\left(\mathrm{a}, T_{c}=45 \mathrm{~K}, x \approx 0.075\right)$ and in $\mathrm{YBa}_{2} \mathrm{Cu}_{3} \mathrm{O}_{6.83}\left(\mathrm{~b}, T_{c}=85 \mathrm{~K}, x \approx 0.14\right.$ ) at $T=5 \mathrm{~K}$. Here and in Fig. 2 tick labels on the vertical axes correspond to the curves. In both figures experimental values are approximately 1.5 times smaller than the calculated ones.

FIG. 2: The imaginary part of the spin susceptibility in the normal state. Curves show the results of our calculations for $T=116 \mathrm{~K}$, all other parameters are the same as for the respective panels in Fig. 11 Filled squares are the odd susceptibility measured ${ }^{2}$ in $\mathrm{YBa}_{2} \mathrm{Cu}_{3} \mathrm{O}_{6.5}$ (a) and in $\mathrm{YBa}_{2} \mathrm{Cu}_{3} \mathrm{O}_{6.83}$ (b) at $T=100 \mathrm{~K}$. 
FIG. 3: The frequency dependence of the total damping $\Gamma(\mathbf{Q})$ used in the calculation of the four curves in Figs. 11 and 2 The parameter $f$ is equal to 2.7 for the dashed curve and 2 for the other curves. The parameter $\eta$ is equal to $0.027 t$, $0.04 t, 0.012 t$, and $0.029 t$ for the solid, dashed, short-dashed and dash-dotted curves, respectively.

relative intensity of this shoulder. The damping $\eta$ can be connected with the processes of multiple spin-excitation scattering or scattering at impurities. The frequency dependencies of the total damping used in the calculation of the curves in Figs. 1 and 2 are shown in Fig. 3 Notice that the fitting parameters $f$ and $\eta$ with the values given in the caption to this figure influence only weakly the position of the maximum in susceptibility which is determined by the value of $\omega_{\mathbf{Q}}$.

As seen from Figs. 1 1 and 2] the position of this maximum, the resonance peak, and its evolution with doping and temperature described by the $t-J$ model are in good agreement with those observed in $\mathrm{YBa}_{2} \mathrm{Cu}_{3} \mathrm{O}_{7-y}$. In the model the maximum is connected with the excitation of localized $\mathrm{Cu}$ spins at the antiferromagnetic wave vector Q. Its frequency $\omega_{\mathbf{Q}}$ determines the size of the spin gap. In the underdoped case it determines also the frequency of the resonance peak. As shown in Ref. 14, $\omega_{\mathbf{Q}}$ grows with doping and this leads to the growth of the frequency of the resonance peak from approximately $18 \mathrm{meV}$ at $x=0.06$ to $38 \mathrm{meV}$ at $x=0.12$ in Figs. 1 and 2 It was also shown ${ }^{14}$ that $\operatorname{Im} \chi(\mathbf{k} \omega)$ is strongly peaked at $\mathbf{Q}$ and that the value of $\operatorname{Im} \chi(\mathbf{Q} \omega)$ decreases with doping which is in agreement with experimental observations 1.2 In absolute units our calculated values of $\operatorname{Im} \chi(\mathbf{Q} \omega)$ are approximately 1.5 times larger than its experimental values.

We notice that the shape of the calculated frequency dependence of the susceptibility is close to that observed experimentally. Of special interest is the low-frequency shoulder in this dependence. This shoulder is more pronounced for lower hole concentrations and temperatures. As mentioned above, it originates from the strong frequency dependence of the damping $\Gamma(\mathbf{Q})$ shown in Fig. 3.
FIG. 4: The Fermi surface of the $t-J$ model (lines) and the momenta which give the main contribution to the maxima of $\Gamma(\mathbf{Q})$ in the used $20 \times 20$ lattice (circles). The antiferromagnetic wave vector $\mathbf{Q}$ connecting momenta of the fermion pair in the spin polarization bubble is shown by the arrow. The point $M$ corresponds to $\mathbf{k}=(\pi, \pi)$.

The pronounced maxima of the curves in this figure are connected with intensive peaks in the hole spectral function for momenta near the Fermi surface. These peaks correspond to the so-called spin-polaron band 15 For moderate doping the Fermi surface of the $t$ - $J$ model consists of two rhombuses with rounded corners 14 These rhombuses are centered at the $\Gamma$ and $M$ points and are approximately nested by the momentum $\mathbf{Q}$. This nesting is also very essential for the appearance of the maximum in $\Gamma(\mathbf{Q} \omega)$. In Fig. 4 the Fermi surface is shown and momenta of the hole spectral functions which give the main contribution to the maxima of $\Gamma(\mathbf{Q})$ in the used $20 \times 20$ lattice are indicated. For these momenta the intensive spin-polaron maxima in the spectral functions $A\left(\mathbf{k}^{\prime} \omega^{\prime}\right)$ and $A\left(\mathbf{Q}+\mathbf{k}^{\prime}, \omega+\omega^{\prime}\right)$ [see Eq. (16)] overlap and fall into the frequency window determined by the difference of the occupation numbers.

The Fermi surface in $\mathrm{YBa}_{2} \mathrm{Cu}_{3} \mathrm{O}_{7-y}$ differs from that shown in Fig. $4^{23.24}$ However, it is known from the photoemission experiments that at least in the superconducting state the hole spectral function has pronounced peaks for momenta near the Fermi surface. In the two-layer $\mathrm{YBa}_{2} \mathrm{Cu}_{3} \mathrm{O}_{7-y}$ the main contribution to the damping of the spin excitations is given by the decay into the fermion pair in which one of the fermions belongs to the bonding band and the other to the antibonding band and the respective parts of the Fermi surface are nested by the momentum $(\pi, \pi, \pi), 9.24$ These conditions are similar to those observed in the $t-J$ model and therefore the lowfrequency shoulder in the susceptibility in $\mathrm{YBa}_{2} \mathrm{Cu}_{3} \mathrm{O}_{7-y}$ can be also related to the strong frequency dependence of the damping of the spin excitations which arises due to pronounced peaks in the hole spectral function and the nesting. 
FIG. 5: Constant energy $(\kappa, \kappa)$ scans at the resonance energy $\omega_{\mathbf{Q}}$. Solid and dashed curves show the results of our calculations for $x=0.12, \omega_{\mathbf{Q}}=38 \mathrm{meV}$ in the superconducting state at $T=17 \mathrm{~K}$ and in the normal state at $T=116 \mathrm{~K}$, respectively. To simulate a finite instrumental momentum resolution the curves were calculated by the convolution of $\operatorname{Im} \chi(\mathbf{k} \omega)$ with the Gaussian with the full width at half maximum equal to $0.2 \pi$ in the momentum space. Filled and open squares are experimental data ${ }^{25}$ in $\mathrm{YBa}_{2} \mathrm{Cu}_{3} \mathrm{O}_{6.83}$ for $\omega_{\mathbf{Q}}=35 \mathrm{meV}$, $T=4 \mathrm{~K}$ and $109 \mathrm{~K}$, respectively.

It is worth noting that for all four curves in Figs. 1 and 2 the value of $\Gamma\left(\mathbf{Q}, \omega_{\mathbf{Q}}\right) / 2$ is smaller than $\omega_{\mathbf{Q}}$. Thus, in contrast to a vicinity of the $\Gamma$ point near the $M$ point the spin excitations are not overdamped in underdoped $\mathrm{YBa}_{2} \mathrm{Cu}_{3} \mathrm{O}_{7-y}$.

Now let us consider the momentum dependence of the resonance mode. In Fig. 5 the constant energy scans obtained in our calculations are compared with experiment ${ }^{25}$ in $\mathrm{YBa}_{2} \mathrm{Cu}_{3} \mathrm{O}_{6.83}$. The scans were performed along the diagonal of the Brillouin zone at the resonance energy in the superconducting and normal states. To simulate a finite instrumental momentum resolution, which is comparable to the width of the peak in $\operatorname{Im} \chi(\mathbf{k} \omega)$ our curves were calculated by the convolution of this quantity with the Gaussian with the full width at half maximum equal to $0.2 \pi$ in the momentum space. This corresponds to 0.1 in reciprocal lattice units which is the usual resolution in experiments of this type. As can be seen from Fig. 5] for both temperatures the calculated momentum dependencies are in good agreement with experiment.

In Fig. 6] the dispersion of the maximum of our calculated susceptibility is compared with experimental data 2 in $\mathrm{YBa}_{2} \mathrm{Cu}_{3} \mathrm{O}_{6.5}$. This dispersion corresponds approximately to $\omega_{\mathbf{k}}$ in Eq. (13). For small $\mathbf{q}=\mathbf{k}-\mathbf{Q}$ this momentum dependence can be written as

$$
\omega_{\mathbf{k}} \approx \sqrt{\omega_{\mathbf{Q}}^{2}+c^{2}(\mathbf{k}-\mathbf{Q})^{2}} .
$$

This function fitted to our calculated data with the parameters $\omega_{\mathbf{Q}}=18.4 \mathrm{meV}$ and $c / a=\sqrt{8 \alpha\left|C_{1}\right|} J=$
FIG. 6: The dispersion of the maximum in the frequency dependence of $\operatorname{Im} \chi(\mathbf{q} \omega), \mathbf{q}=\mathbf{k}-\mathbf{Q}$. Filled squares are our results for $x=0.06$ and $T=17 \mathrm{~K}$. The fit for these data with Eq. (17) is shown by the curve. Open squares are experimental results ${ }^{2}$ in $\mathrm{YBa}_{2} \mathrm{Cu}_{3} \mathrm{O}_{6.5}$ at $T=5 \mathrm{~K}$ for odd spin excitations.

$0.134 \mathrm{meV}$ is also shown in Fig. 6] Here $a$ is the distance between $\mathrm{Cu}$ sites in a $\mathrm{Cu}-\mathrm{O}$ plane. As seen from this figure, our calculated dispersion is close to the experimental one for similar parameters. For $\omega \approx \omega_{\mathbf{Q}} \operatorname{Im} \chi(\mathbf{k} \omega)$ is peaked at $\mathbf{k}=\mathbf{Q}$. For $\omega>\omega_{\mathbf{Q}}$ the susceptibility has maxima on the ring with the radius approximately determined by the equation $\omega=\left[\omega_{\mathbf{Q}}^{2}+c^{2}(\mathbf{k}-\mathbf{Q})^{2}\right]^{1 / 2}$. In constant energy scans along some direction this property of the susceptibility manifests itself as two peaks in incommensurate positions equally spaced from the $M$ point ${ }^{26}$ For $\omega<\omega_{\mathbf{Q}}$ for the considered parameters $\operatorname{Im} \chi(\mathbf{k} \omega)$ is peaked at $\mathbf{k}=\mathbf{Q}$.

\section{CONCLUDING REMARKS}

We have considered the magnetic susceptibility for the underdoped case when the resonance peak is observed both in the normal and in the superconducting states. As mentioned, the frequency of the peak is determined by the frequency of the spin excitation $\omega_{\mathbf{Q}}$ which sets the size of the spin gap. This frequency grows with the hole concentration, 14 in agreement with experimental observations in underdoped crystals. 2.6

For the normal-state $t-J$ model in the overdoped region it was shown ${ }^{27}$ that the part of the magnon branch, which persisted at lower doping at the periphery of the Brillouin zone, is suddenly destroyed for $x \approx 0.17$ at $T=0$. This transition is accompanied by the radical change of the hole spectrum: dispersion and distribution of the spectral weight become close to the case of weakly correlated fermions. This result corresponds to the sudden disappearance of the resonance peak in the normal-state overdoped cuprates ${ }^{2,6}$ One of the reasons for the transition 
in the $t$ - $J$ model is the damping of the spin excitations which grows with doping. A considerable decrease of the damping in the superconducting state can restore the spin excitations near the $M$ point in the frequency range $\omega \leq 2 \Delta^{s}$. Such mechanism was considered in Ref. 11 where the magnetic susceptibility similar to that given by Eqs. (12) and (13) was postulated and the damping described by the fermion bubble of the type of Eq. (16) was used. Above the mentioned transition at $x \approx 0.17$ the hole spectrum of the $t-J$ model becomes similar to that used in Ref. 11 and the analogous outcome can be expected here.

In contrast to the underdoped region, in the overdoped case the frequency of the resonance peak decreases with doping which can be related to a finite damping of the spin excitations and to the decrease of the superconducting gap with doping in this range of concentrations ${ }^{21}$

In contrast to $\mathrm{YBa}_{2} \mathrm{Cu}_{3} \mathrm{O}_{7-y}$ where $\omega_{\mathbf{Q}} \lesssim 2 \Delta^{s}$, in $\mathrm{La}_{2-x} \mathrm{Sr}_{x} \mathrm{CuO}_{4}$ the value of $2 \Delta^{s} \approx 9 \mathrm{meV}$ is substantially smaller than $\omega_{\mathbf{Q}}$ which is supposed to be approximately the same as in the former crystal. This difference may be the reason for the absence of the resonance peak in overdoped $\mathrm{La}_{2-x} \mathrm{Sr}_{x} \mathrm{CuO}_{4} \stackrel{11}{=}$ Changes in the susceptibility observed ${ }^{28}$ in $\mathrm{La}_{1.86} \mathrm{Sr}_{0.14} \mathrm{CuO}_{4}$ at the superconducting transition consist of some suppression of $\operatorname{Im} \chi$ below the superconducting gap and an increase above it. The suppression can be connected with the decrease of the damping of the spin excitation accompanying the open- ing of the gap, while the increase of the signal above the gap is apparently a combined effect of the transfer of the carrier spectral weight above the gap and the nesting supposed $^{29}$ for the Fermi surface of this crystal.

In conclusion, we compared the magnetic susceptibility calculated in the $t-J$ model with the experimental data in the underdoped $\mathrm{YBa}_{2} \mathrm{Cu}_{3} \mathrm{O}_{7-y}$. It was demonstrated that the calculations reproduce correctly the frequency and momentum dependencies of the experimental susceptibility and its variation with doping and temperature in the normal and superconducting states. This allowed us to interpret the maximum in the frequency dependence - the resonance peak - as a manifestation of the excitation branch of localized $\mathrm{Cu}$ spins and to relate the frequency of the maximum to the size of the spin gap. The low-frequency shoulder well resolved in the susceptibility of superconducting crystals was connected with a pronounced maximum in the damping of the spin excitations. This maximum is caused by intense quasiparticle peaks in the hole spectral function for momenta near the Fermi surface and by the nesting.

\section{Acknowledgments}

This work was partially supported by the ESF grant No. 5548 and by DFG.
1 J. Rossat-Mignot, L. P. Regnault, P. Bourges, P. Burlet, C. Vettier, and J. Y. Henry, in Selected Topics in Superconductivity, edited by L. C. Gupta and M. S. Multani (World Scientific, Singapore, 1993), p. 265.

2 P. Bourges, in The Gap Symmetry and Fluctuations in High Temperature Superconductors, edited by J. Bok, G. Deutscher, D. Pavuna, and S. A. Wolf (Plenum Press, 1998), p. 349.

3 J. Rossat-Mignot, L. P. Regnault, C. Vettier, P. Bourges, P. Burlet, J. Bossy, J. Y. Henry, and G. Lapertot, Physica C 185-189, 86 (1991).

${ }^{4}$ H. A. Mook, M. Yethiraj, G. Aeppli, T. E. Mason, and T. Armstrong, Phys. Rev. Lett. 70, 3490 (1993).

${ }^{5}$ H. F. Fong, B. Keimer, D. L. Milius, and I. A. Aksay, Phys. Rev. Lett. 78, 713 (1997); P. Dai, H. A. Mook, and F. Doğan, Phys. Rev. Lett. 80, 1738 (1998).

${ }^{6}$ H. He, Y. Sidis, P. Bourges, G. D. Gu, A. Ivanov, N. Koshizuka, B. Liang, C. T. Lin, L. P. Regnault, E. Schoenherr, and B. Keimer, Phys. Rev. Lett. 86, 1610 (2001); P. Bourges, B. Keimer, S. Pailhès, L. P. Regnault, Y. Sidis, and C. Ulrich, cond-mat/0211227 (unpublished)

7 D. Z. Liu, Y. Zha, and K. Levin, Phys. Rev. Lett. 75, 4130 (1995).

8 I. I. Mazin and V. M. Yakovenko, Phys. Rev. Lett. 75, 4134 (1995).

9 N. Bulut and D. J. Scalapino, Phys. Rev. B 53, 5149 (1996).

10 A. Abanov and A. V. Chubukov, Phys. Rev. Lett. 83, 1652 (1999).
11 D. K. Morr and D. Pines, Phys. Rev. Lett. 81, 1086 (1998).

12 M. A. Kastner, R. J. Birgeneau, G. Shirane, and Y. Endoh, Rev. Mod. Phys. 70, 897 (1998).

13 J. H. Jefferson, H. Eskes, and L. F. Feiner, Phys. Rev. B 45, 7959 (1992); A. V. Sherman, Phys. Rev. B 47, 11521 (1993).

14 A. Sherman and M. Schreiber, Phys. Rev. B 65, 134520 (2002); European Phys. J. B 32, 203 (2003).

15 Yu. A. Izyumov, Usp. Fiz. Nauk 167, 465 (1997) [Phys.Usp. (Russia) 40, 445 (1997)]; E. Dagotto, Rev. Mod. Phys. 66, 763 (1994).

16 J. Kondo and K. Yamaji, Progr. Theor. Phys. 47, 807 (1972); H. Shimahara and S. Takada, J. Phys. Soc. Jpn. 60, 2394 (1991).

17 N. D. Mermin and H. Wagner, Phys. Rev. Lett. 17, 1133 (1966).

18 A. Sherman and M. Schreiber, in Studies of High Temperature Superconductors, edited by A. V. Narlikar (Nova Science Publishers, New York, 1999), vol. 27, p. 163; Physica C 303, 257 (1998).

19 D. Forster, Hydrodynamic Fluctuations, Broken Symmetry, and Correlation Functions (W. A. Benjamin, Inc., London, 1975).

20 A. K. McMahan, J. F. Annett, and R. M. Martin, Phys. Rev. B 42, 6268 (1990); V. A. Gavrichkov, S. G. Ovchinnikov, A. A. Borisov, and E. G. Goryachev, Zh. Eksp. Teor. Fiz. 118, 422 (2000) [JETP (Russia) 91, 369 (2000)].

21 N.-C. Yeh, C.-T. Chen, R. P. Vasquez, C. U. Jung, S.I. Lee, K. Yoshida, and S. Tajima, J. Low Temp. Phys. 
131, 435 (2003)

22 J. L. Tallon, C. Bernhard, H. Shaked, R. L. Hitterman, and J. D. Jorgensen, Phys. Rev. B 51, 12911 (1995).

23 A. Damascelli, Z. Hussain, and Z.-X. Shen, Rev. Mod. Phys. 75, 473 (2003).

${ }^{24}$ O. K. Andersen, O. Jepsen, A. I. Liechtenstein, and I. I. Mazin, Phys. Rev. B 49, 4145 (1994).

25 P. Bourges, L. P. Regnault, J. Y. Henry, C. Vettier, Y. Sidis, and P. Burlet, Physica B 215, 30 (1995).

${ }^{26}$ H. F. Fong, P. Bourges, Y. Sidis, L. P. Regnault, J. Bossy,
A. Ivanov, D. L. Milius, I. A. Aksay, and B. Keimer, Phys. Rev. B 61, 14773 (2000).

27 A. Sherman, Phys. Rev. B 55, 582 (1997).

28 T. E. Mason, A. Schröder, G. Aeppli, H. A. Mook, and S. M. Hayden, Phys. Rev. Lett. 77, 1604 (1996).

29 Q. Si, Y. Zha, K. Levin, and J. P. Lu, Phys. Rev. B 47, 9055 (1993); P. Bénard, L. Chen, and A.-M. S. Tremblay, Phys. Rev. B 47, 15217 (1993). 


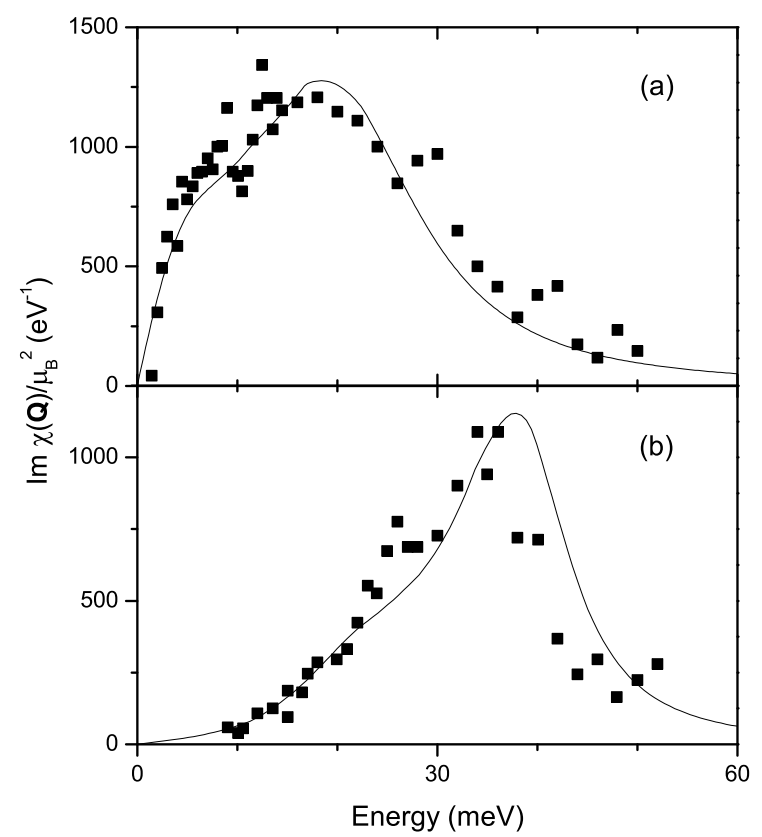




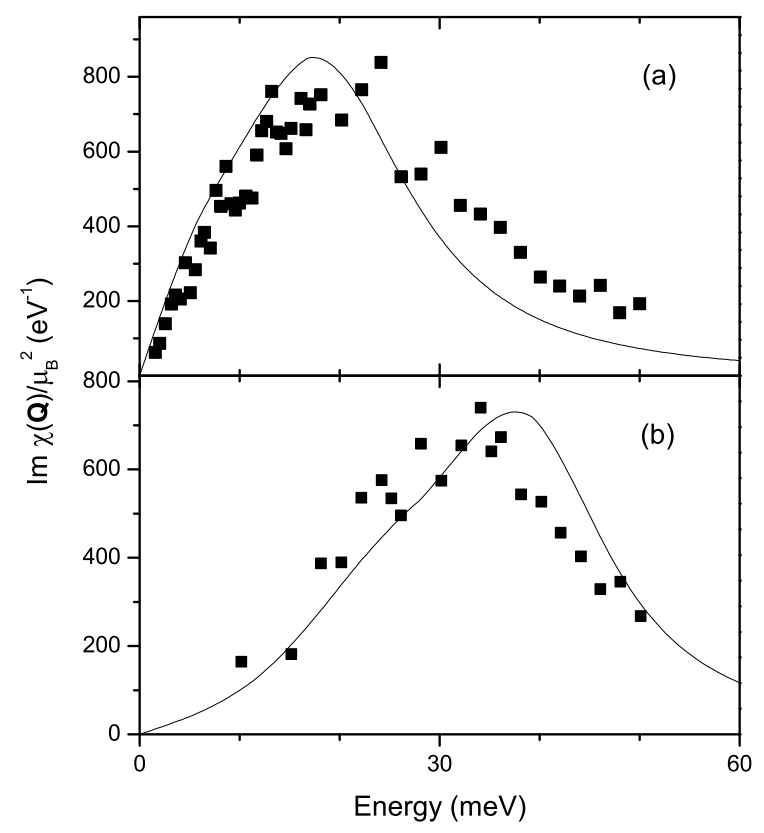




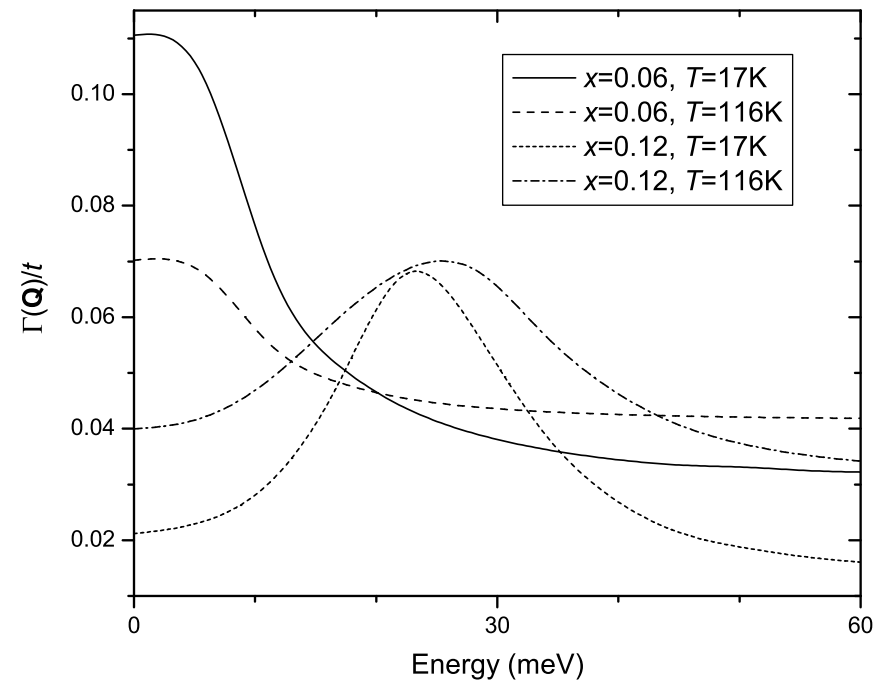



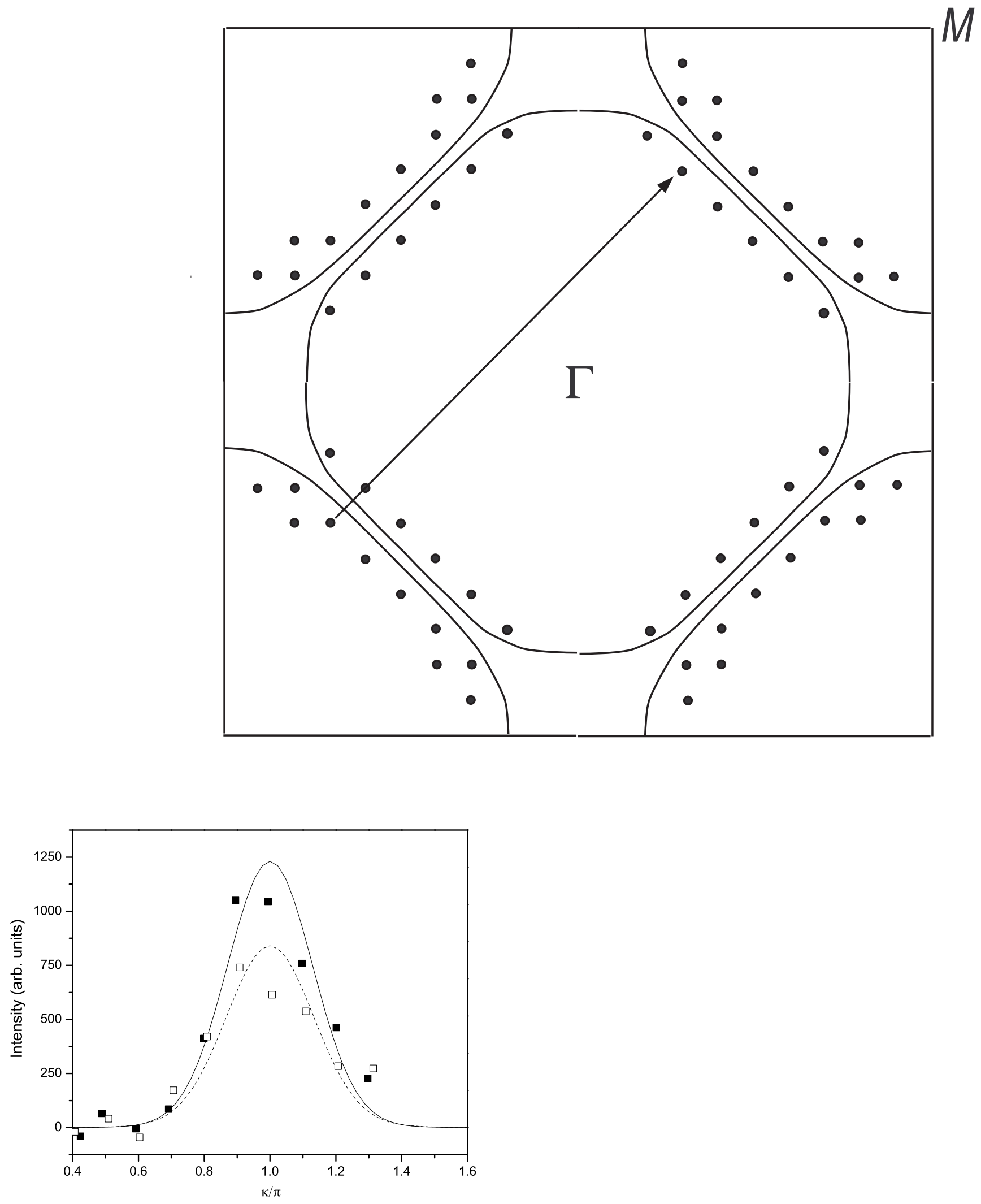


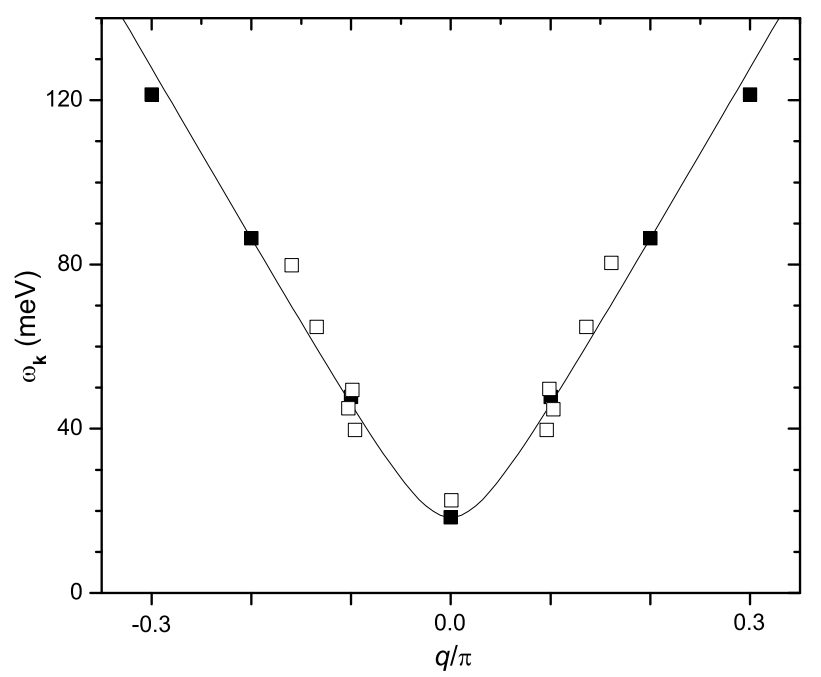

